\title{
The Effect of Using Olive Oil and Fish Oil Prophylactic Dressings on Heel Pressure Injury Development in Critically III Patients
}

This article was published in the following Dove Press journal: Clinical, Cosmetic and Investigational Dermatology

\author{
Zohreh Karimi' \\ Ali Mousavizadeh ${ }^{2}$ \\ Hossein Rafiei ${ }^{3}$ \\ Naeem Abdi ${ }^{4}$ \\ Mohammad \\ Behnammoghadam ${ }^{5}$ \\ Maryam Khastavaneh (D) $^{6}$ \\ Sharif Shahini $\mathbb{D}^{6}$ \\ 'Department of Operating Room, School \\ of Paramedicine, Yasuj University of \\ Medical Sciences, Yasuj, Iran; ${ }^{2}$ Social \\ Determinants of Health Research Center, \\ Yasuj University of Medical Sciences, \\ Yasuj, Iran; ${ }^{3}$ Social Determinants of \\ Health Research Center, Research \\ Institute for Prevention of Non- \\ Communicable Diseases, Qazvin \\ University of Medical Sciences, Qazvin, \\ Iran; ${ }^{4}$ School of Paramedicine, Yasuj \\ University of Medical Sciences, Yasuj, \\ Iran; ${ }^{5}$ Medicinal Plants Research Center, \\ Yasuj University of Medical Sciences, \\ Yasuj, Iran; ${ }^{6}$ Department of Nursing, \\ Yasuj University of Medical Sciences, \\ Yasuj, Iran
}

Introduction and Aim: Prevention of pressure injuries in patients hospitalized in intensive care units is significantly important. Therefore, in the present study, the effect of using olive oil and fish oil prophylactic dressings on the development of heel pressure injuries was investigated.

Methods: The present study was a clinical trial conducted in the intensive care unit of Shahid Beheshti Hospital, in Yasuj. Fifty patients, who were at moderate to high risk of pressure injuries development, were randomly divided into two groups based on the mean score of the Braden scale. In one group, patients' heels were dressed using olive oil prophylactic dressing, and in the other group, patients' heels were dressed using fish oil prophylactic dressing. The dressings were changed 3 times a day. Collected data were then analyzed using SPSS v16.

Results: No significant difference was determined in demographic variables among the two groups ( $\mathrm{p}<0.05$ ). In terms of the development of heel pressure injuries, none of the patients in the olive oil and fish oil groups had pressure injuries.

Conclusion: There were no statistically significant differences in either treatment group related to heel pressure injuries outcomes during the 7 days observed in the study. Additionally, both dressings had the same effects. Further studies are recommended in this regard.

Keywords: preventive dressings, pressure injuries, nursing care, critical patients, olive oil, fish oil

\section{Introduction}

Despite the significant development in the prevention of pressure injuries, many hospitalized patients, especially those hospitalized in intensive care units (ICUs), develop some degrees of pressure injuries. ${ }^{1,2}$ These ulcers can be associated with various issues for the patient and the healthcare system including an increase in the costs, whether during hospitalization or after hospital discharge, an increase in the length of stay in hospital, and an increase in the need for readmission. ${ }^{3}$ In addition, the development of pressure injuries in a patient can lead to psychological problems, lower self-esteem, and lower quality of life. ${ }^{4,5}$ Excessive pressure on the capillaries of the skin and their blockage are the main causes of pressure injuries development. ${ }^{6}$ However, other factors, including immobility, nutritional deficiencies, old age, the frequent use of medical equipment. ${ }^{7}$, urinary and/or bowel incontinence, loss of consciousness, sedation, and some underlying diseases increase the chance of patients developing pressure injuries. ${ }^{8-13}$
Correspondence: Mohammad Behnammoghadam

Medicine Plants Research Center, Yasuj University of Medical Sciences, Next to Imam Sajad Hospital, Bagher Street, Yasuj, Iran

Tel +987433236172

Fax +98743323401 I

Email mbehnam1363@gmail.com 


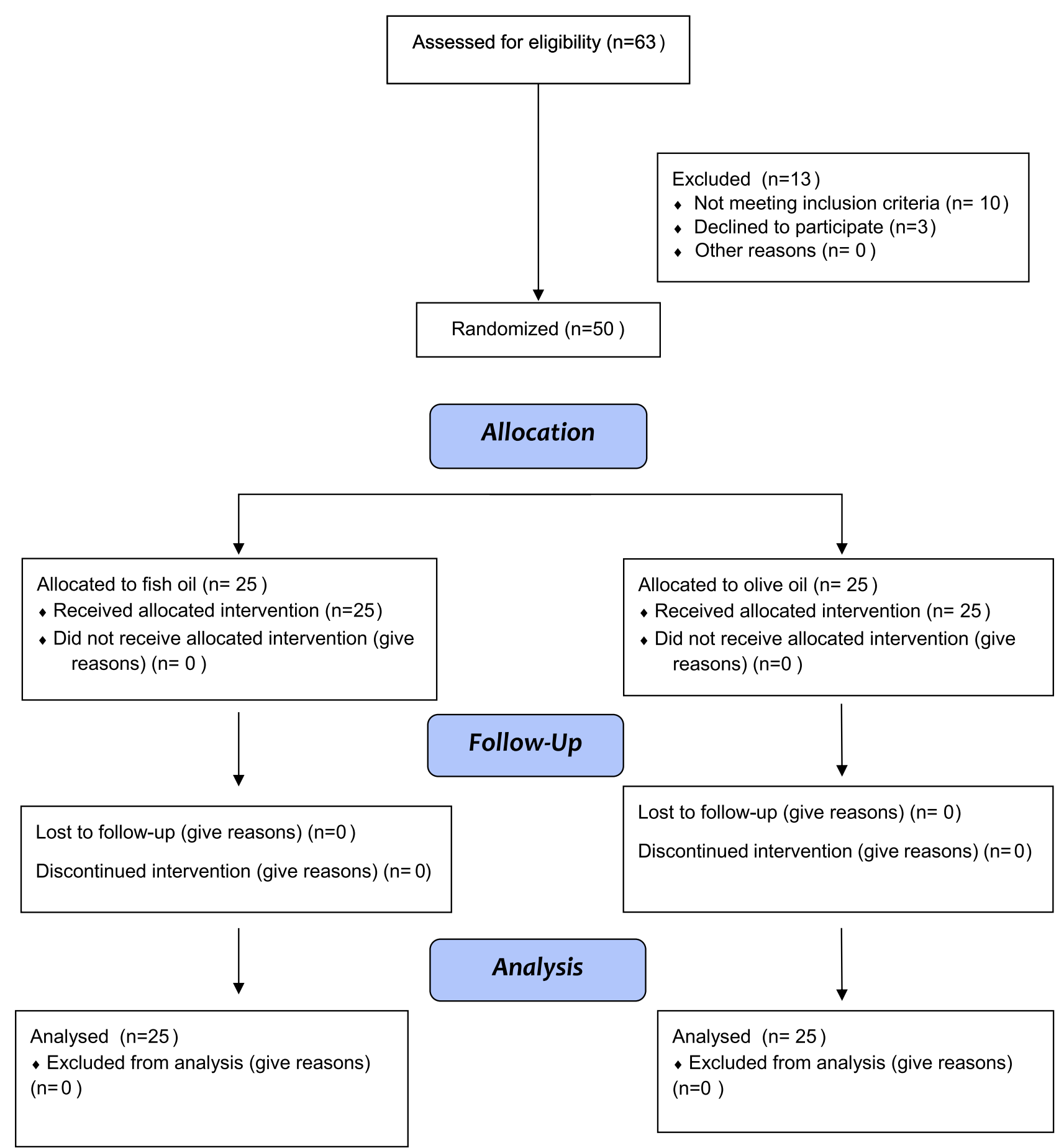

Figure I Consort flow diagram.

Pressure injuries can occur in different parts of the body, especially over the bony areas. One of these areas, which is at relatively high risk of pressure injuries development, is heel. In some sources, heel has been addressed as the second most common part of pressure injuries development. ${ }^{14}$ Based on this, one of the recommendations for preventing such ulcers is the use of prophylactic dressings. ${ }^{15}$ These dressings can generally be divided into three categories: Film, Hydrocolloid, and Foam dressings. ${ }^{16}$ Several studies have conducted in this regard. For instance, in a study in 2017, Teo et al evaluated the effectiveness of prophylactic dressings in preventing heel pressure injuries among 326 patients hospitalized in ICU. For the patients in the intervention group, Teo et al used 
a five-layer foam dressing. The results of this study showed that the use of such dressings significantly reduced the likelihood of pressure injuries development in the heel. ${ }^{14}$ In another study, Santamaria et al reported that the use of multilayered foam dressings in critical patients significantly reduced the development of pressure injuries in the heel and sacrum. ${ }^{17}$

Most of the prophylactic dressings available on the market are industrial ones which are executively produced by some of the manufacturing companies in developed countries. Because of this, there are some barriers to use such dressings. One of the barriers in developing countries such as Iran is the restriction of their financial resources to purchase such dressings. Additionally, many of these dressings are not even available in developing countries including Iran. Due to the relatively high cost of industrial prophylactic dressings and the unavailability of some of them, the use of non-industrial and alternative ones has increased in recent years. Two of the alternative products used in previous studies were olive oil and oils containing fatty acids such as fish oil. The fatty acids and vitamins available in these oils have protective effects on the skin. ${ }^{18,19}$ In a study in this regard, Behnammoghadam et al investigated the effect of local olive oil on different parts of the body and its effect on the prevention of pressure injuries in a group of patients hospitalized in the ICU. The results of this study showed that the use of local olive oil reduced the risk of pressure injuries development by about $15 \% .{ }^{18}$ In another clinical trial, Aloweni et al evaluated the effect of silicone foam and fatty acids oil dressings on the prevention of pressure injuries. The results of this study showed that both types of dressings had better preventive effects compared to the standard care among very high-risk patients. The results also showed no difference between the two types of dressings (silicone foam and fatty acid oil dressings) in the prevention of pressure injuries. ${ }^{20}$

Although the effectiveness of olive oil dressings has been proved to some extent in previous studies, most of these studies have used this oil alone. Furthermore, the preventive effect of olive oil dressing has been less studied with other products so far. In addition, the effectiveness of some oils containing fatty acids, such as fish oil, has not been well studied and studies in this regard are limited. Therefore, the present study was designed and conducted with three main objectives: (1) to evaluate the effectiveness of olive oil prophylactic dressings in the prevention of heel pressure injuries, (2) to evaluate the effectiveness of fish oil prophylactic dressings in the prevention of heel pressure injuries, and (3) to compare the effectiveness of olive oil and fish oil prophylactic dressings in the prevention of heel pressure injuries.

\section{Methods \\ Study Setting}

The proposal of the present clinical trial was reviewed and approved at Yasuj University of Medical Sciences, in 2018. The study was conducted in 2019. The study setting was the ICU of Shahid Beheshti Hospital, in Yasuj. The ICU of this hospital contains 15 beds, and surgical, medical, and trauma patients were hospitalized in it. The incidence of heel pressure injuries in the ICU of this hospital was determined in the year before the study by the Hospital Infection Control about $6 \%$. In this unit, no specific protocol is used to prevent heel pressure injuries, and examining the skin at each shift and changing the patient's position based on the patient's need are the two main routine and nursing care.

\section{Sampling}

In the present study, sampling was done randomly. For this purpose, at the beginning of the study, 12 eligible patients, who were hospitalized in the unit, were divided into two groups using the fishbowl technique. This was done by one of the nurses working in the hospital who did not have any information about the study and its objectives. Then, each new patient, who was hospitalized in the unit after the first sampling, was randomly allocated to one of the two groups using a random number table after signed written informed consent by family (Figure 1).

The results of Karimi et al study ${ }^{27}$ into Impact of olive oil and honey on the healing of diabetic foot: a randomized controlled trial on the variables, as the most similar variables to the dependent variables of the present study were used in the formula calculation.

According to the following formula and considering 95\% confidence level (for mean difference), $80 \%$ test power and the least significant difference, the number of samples required for the study was considered 25 for each group.

$$
\mathrm{n}=\frac{2\left[\left(\mathrm{z}_{1-\frac{\alpha}{2}}+\mathrm{z}_{1-\beta}\right)^{2}\right]\left(\mathrm{s}_{1}^{2}+\mathrm{s}_{2}^{2}\right)}{\left(\mu_{1}-\mu_{2}\right)^{2}}
$$

\section{Inclusion and Exclusion Criteria}

Inclusion criteria for the study were having moderate to high risk of pressure injuries development based on 
a score obtained in the Braden Scale, aged at least 18 years old, and having no sign of pressure injuries in the heel at the time of hospitalization in the ICU. Those patients who were allergic to oils, according to the patient's family and physician's reports, were excluded.

\section{Intervention}

\section{Patients in the Olive Oil Group}

Patients in this group, in addition to the routine care, were treated with a simple gauze soaked in $4 \mathrm{cc}$ of olive oil in both heels daily. This procedure was continued for up to 7 days for each patient. Dressing was performed by one of the researchers with the help of nurses. The heels' skin was evaluated before each dressing using the American Pressure injury Grading Tool. For this purpose, the dressing was first removed by the nurse responsible for the care of the patient and the researcher, and then, the skin was washed. Another researcher, who was unaware of the type of dressing, evaluated the skin in terms of pressure injuries development. The olive oil used in the present study was produced by Loyeh Company in Iran.

\section{Patients in the Fish Oil Group}

Patients in this group, in addition to the routine care, were treated with a simple gauze soaked in $4 \mathrm{cc}$ of fish oil in both heels daily. This procedure was continued for up to 7 days for each patient. Dressing was performed by one of the researchers with the help of nurses. The heels' skin was evaluated before each dressing using the American Pressure injury Grading Tool. For this purpose, the dressing was first removed by the nurse responsible for the care of the patient and the researcher, and then, the skin was washed. Another researcher, who was unaware of the type of dressing, evaluated the skin in terms of pressure injuries development. The fish oil used in the present study was produced by island Fish Processing Company

\section{Data Collection Tools}

In this study, three data collection tools were used:

1. A checklist for demographic variables:

This researcher-made checklist included items about patients' age, gender, reason for hospitalization, the Braden Scale score, and the Glasgow Coma Scale score.

2. Braden Scale for Predicting Pressure injury Risk:

The Braden Scale was used to identify people at moderate to high risk of pressure injuries development. This scale assesses six criteria: Sensory perception, Moisture, Activity, Mobility, Nutrition, Friction and Shear. ${ }^{2}$ Patients with a Braden Scale score of less than 14 were selected and included in the study.

3. American Pressure injury Grading Tool:

This tool was used to identify and stage pressure injuries. Based on this tool, pressure injuries are divided into six stages in terms of its severity. ${ }^{21}$

\section{Ethical Considerations}

This study was conducted in accordance with the Declaration of Helsinki. The present study was conducted under the supervision of Yasuj University of Medical Sciences. The proposal of the study was approved by the Ethics Committee of the University and obtained the ethics code (code: IR.yums.rec.1396.69). The study protocol was also recorded in the Iranian Registry of Clinical Trials. Because patients' level of consciousness were low, a written consent was obtained from the patient's family caregiver before including in the study. To meet this goal, they were given necessary explanations regarding the type of intervention, the amount and duration of the intervention. Those patients who were allergic to oils, according to the patient's family and physician's reports, were excluded. Confidentiality regarding the patients' personal information was also considered as much as possible by the researchers in the present study.

\section{Data Analysis}

Collected data were analyzed using SPSS v16 by a statistician. The statistician did not have any information about patients' groups in the SPSS file. Data analysis had a normal distribution. To compare the mean score of quantitative variables between the two groups, an independent $t$-test was used considering the significance level of less than 0.05 . To compare the qualitative variables between the two groups including gender, chi-square test was used.

\section{Results}

No adverse reactions were observed in the patients' heels in the two groups because of using olive and fish oil. All the demographic variables recorded were compared between the two groups. Only in the nutrition section of the Braden Scale, there was a difference between the patients, in which the score in the fish oil group was slightly higher than in the olive oil group. However, there was no significant difference in the mean overall score of the Braden Scale among the two groups. No significant difference was determined between the other patients' demographic variables among the two groups. 
Table I Patients' Demographic Characteristics

\begin{tabular}{|l|l|l|l|l|}
\hline \multicolumn{2}{|l|}{ Item } & Olive Oil & Fish Oil & P value \\
\hline \multirow{2}{*}{ Sex } & Male & $\mathrm{II}$ & 13 & 0.374 \\
\cline { 2 - 4 } & Female & 9 & 7 & \\
\hline Age & $41.1 \pm 12.3$ & $45.2 \pm 13.8$ & 0.323 \\
\hline GCS & $7.3 \pm 1.8$ & $6.96 \pm 2.3$ & 0.062 \\
\hline Body mass index & $26.5 \pm 3.6$ & $27.7 \pm 5.4$ & 0.431 \\
\hline Sensory perception & 2.45 & 2.30 & 0.457 \\
\hline Moisture & 1.75 & 1.55 & 0.316 \\
\hline Mobility & 1 & 1 & 0.471 \\
\hline Activity & 1 & 1 & 0.471 \\
\hline Nutrition & 1.50 & 1.20 & 0.048 \\
\hline Friction and shear & 1.50 & 1.30 & 0.06 \\
\hline Total Braden score & $9.2 \pm 1.9$ & $8.3 \pm 1.3$ & 0.083 \\
\hline
\end{tabular}

Table 1 illustrates this in more detail. According to the Braden Scale scores, all the patients in the present study were at high risk of pressure injuries development. Rate of pressure injuries development were zero in patients in both groups.

\section{Discussion}

Pressure injuries in patients hospitalized in the ICUs can be a major challenge for the patient and the healthcare team, especially the nursing staff. Regarding this, in response to questions number one and two in the introduction, the results of the present study surprisingly showed that the use of olive oil and fish oil prophylactic dressings prevented pressure injuries development. In response to question number three in the introduction, the results also showed that both types of dressings had the same effects on the prevention of pressure injuries in the heels in patients hospitalized in the ICU.

The use of oils containing fatty acids in the prevention of pressure injuries has been of great interest in recent years. One of these oils is olive oil. The results of this study approved the preventive effect of olive oil dressings on pressure injuries development. The results of previous studies in this regard are slightly different from the findings of the present study, which can be due to several reasons. For instance, in a study in Iran in 2015, Abbas Ali Madadi et al investigated the effect of using topical olive oil on the prevention of pressure injuries in ICUs patients. ${ }^{22}$ In this study, the results showed that the use of this oil significantly reduced the development of pressure injuries in hospitalized patients (the development of ulcers in patients who received oil was $16 \%$ and in those who received the routine care was $40 \%)$. However, the development of pressure injuries in the olive oil group in the present study was zero, which is much lower than the $16 \%$ reported in that study. In another study, Behnammoghadam et al reported that topical use of olive oil on the patients' skin significantly reduced the development of pressure injuries in them. ${ }^{18}$ However, in the study of Behnammoghadam et al, the development of pressure injuries in the intervention group receiving olive oil was $14 \%$ which was higher than which in the present study. There are three reasons for the high development of pressure injuries in the studies of Behnammoghadam et al and Abbas Ali Madadi et al. Firstly, in the studies of Behnammoghadam et al and Abbas Ali Madadi et al, olive oil was only rubbed on the skin of the patients, whereas in the present study, a prophylactic dressing impregnated with olive oil was applied, which it can be effective in reducing the pressure on the skin. Secondly, in the present study, the development of pressure injuries was evaluated in the patients' heels, whereas the two mentioned studies investigated the effect of olive oil on the development of pressure injuries in all parts of the body. Lastly, the duration of the intervention among the studies was different. The duration of the intervention in the present study was 7 days, while in the two mentioned studies, the intervention was continued until the discharge, and as it is clear, an increase in the length of stay in hospital can significantly increase the risk of pressure injuries development in hospitalized patients.

Our searches did not reveal a study on the use of fish oil in the prevention of pressure injuries. However, the searches showed a study that had evaluated the therapeutic effect of this oil on stage 1 pressure injuries in patients hospitalized in the ICUs. In this study conducted in 2012, 60 patients with stage 1 pressure injuries in the sacrum, heels and shoulders were divided into two groups. For patients in the intervention group in this study, topical fish oil was applied every 2 to $3 \mathrm{hrs}$. This intervention was continued for 7 days. If they improved, the method was stopped in the first days of the procedure. Finally, the results of this study showed that the use of fish oil significantly accelerated the process of healing of these ulcers. ${ }^{23}$ It was also reported in a case report that the use of fish oil-derived fats (Omega-3 acid ethyl esters) was effective in treating pressure injuries in a 21-year-old female patient. ${ }^{24}$ 
The results of the present study also approved the preventive effects of olive oil and fish oil dressings on the prevention of pressure injuries in the heels in patients hospitalized in the ICU. Our searches did not show a study that compared the two oils as in the present study. However, a study in 2017 compared the effect of olive oil with hyper-oxygenated fatty acids on the prevention of pressure injuries. The results of this study showed that the two products had relatively similar effects on the prevention of pressure injuries in the sacrum and heels. ${ }^{25}$ Using fish oil can be helpful in many skin problems, such as skin allergies, dermatitis, skin changes due to aging, skin problems related to cancers and cutaneous wounds. The skin's protective properties of this oil appear to be due to the presence of omega-3 polyunsaturated fatty acids, docosahexaenoic acid, eicosapentaenoic acid, $\alpha$-linolenic acid, and linoleic acid. ${ }^{19}$ It also contains some vitamins, such as vitamins $\mathrm{A}$ and $\mathrm{E}$, which have protective effects on the skin. ${ }^{23}$ On the other hand, a significant portion of olive oil is composed of oleic acid, which plays a significant role in restoring and maintaining moisture and skin freshness. In addition, vitamin $\mathrm{E}$ in this oil also has protective effects on the skin. ${ }^{26}$ These properties in olive oil appear to be effective in preventing pressure injuries development in the heels. However, in future studies, the mechanism of action of olive oil and fish oil needs to be studied in greater detail.

\section{Limitations}

We have no control group and its main limitation for our study. The duration of the intervention in the present study was 7 days. This may be considered as a limitation and it is recommended that patients be examined for a longer period in future studies. Also, in the present study dressing was used only in the heels. In future studies, it is recommended that these dressings be used in other parts of the body, such as sacrum and occiput. Most patients in our study were young and adults, and its limited generability of our finding to pediatrics, neonatal and older patients admitted to ICU.

\section{Conclusions}

The prevention of pressure injuries in patients hospitalized in ICUs is significantly important. In this regard, the results of the present study showed that there were no statistically significant differences in either treatment group related to heel pressure injuries outcomes during the 7 days observed in the study. Additionally, both dressings had the same effects. The results of the present study, if confirmed in future studies, can play a significant role in the prevention of pressure injuries in patients hospitalized in ICUs, especially in countries with moderate to poor economic status.

\section{Data Sharing Statement}

Raw data are available if requested up to 1 year after publishing.

\section{Ethics and Registration}

Ethics committee reference number: IR.yums.rec.1396.69. IRCT registration number: IRCT20180227038885N1.

\section{Acknowledgments}

The present study is part of a larger study approved at Yasuj University of Medical Sciences. We would like to thank the officials of this university. We also thank the patients, the patients' families, the nurses, the head nurse and the physicians of the ICU of Shahid Beheshti Hospital, in Yasuj, who help us conduct this study.

\section{Disclosure}

The authors report no funding and no conflicts of interest in this work.

\section{References}

1. Zarei E, Madarshahian E, Nikkhah A, Khodakarim S. Incidence of pressure ulcers in intensive care units and direct costs of treatment: evidence from Iran. J Tissue Viability. 2019;28(2):70-74.

2. Iranmanesh S, Rafiei H, Sabzevari S. Relationship between Braden scale score and pressure ulcer development in patients admitted in trauma intensive care unit. Int Wound J. 2012;9(3):248-252. doi:10.1111/iwj.2012.9.issue-3

3. Han Y, Jin Y, Jin T, Lee SM, Lee JY. Impact of pressure injuries on patient outcomes in a Korean hospital: a case-control study. $J$ Wound Ostomy Continence Nurs. 2019;46(3):194-200. doi:10.1097/WON. 0000000000000528

4. Galhardo VA, Garroni Magalhaes M, Blanes L, Juliano Y, Masako Ferreira L. Health-related quality of life and depression in older patients with pressure ulcers. Wounds. 2010;22(1):20-26.

5. Lourenco L, Blanes L, Salomé GM, Ferreira LM. Quality of life and self-esteem in patients with paraplegia and pressure ulcers: a controlled cross-sectional study. J Wound Care. 2014;23(6):331-4, 336-7. doi:10.12968/jowc.2014.23.6.331

6. Souza S, Danski MTR, Johann DA, De Lazzari LSM, Mingorance P. Prevention's pressure ulcers heel with transparent polyurethane film. Acta Paul Enferm. 2013;26(4):345-352. doi:10.1590/S0103-21002 013000400008

7. Tafti AA, Sajadi S, Rafiei H. Pressure ulcer stage IV caused by cervical collar in patients with multiple trauma in intensive care unit. Int Wound J. 2015;12(5):606-607. doi:10.1111/iwj.12158.

8. Senmar M, Azimian J, Rafiei H, Habibollahpour M, Yousefi F. The incidence of pressure ulcer in old patients undergoing open heart surgery and the relevant factors. J Prev Epidemiol. 2017;2(2):e09.

9. Bereded DT, Salih MH, Abebe AE. Prevalence and risk factors of pressure ulcer in hospitalized adult patients; a single center study from Ethiopia. BMC Res Notes. 2018;11(1):847. doi:10.1186/s13104-0183948-7 
10. Liao X, Ju Y, Liu G, Zhao X, Wang Y, Wang Y. Risk factors for pressure sores in hospitalized acute ischemic stroke patients. J Stroke Cerebrovasc Dis. 2019;28(7):2026-2030. doi:10.1016/j. jstrokecerebrovasdis.2019.02.033

11. Koo M, Sim Y, Kang I. Risk factors of medical device-related pressure ulcer in intensive care units. J Korean Acad Nurs. 2019;49 (1):36-45. doi:10.4040/jkan.2019.49.1.36

12. Mehta C, Ali M, Mehta Y, George JV, Singh MK. MDRPU -an uncommonly recognized common problem in ICU: a point prevalence study. J Tissue Viability. 2019;28(1):35-39. doi:10.1016/j. jtv.2018.12.002

13. Lucchini A, Elli S, Bianchi F, et al. Incidenza e fattori di rischio associati allo sviluppo di lesionida pressione in una terapia intensiva generale italiana. [Incidence and risk factors associated with the development of pressure ulcers in an Italian general intensive care unit]. Assist Inferm Ric. 2018;37(4):181-188. Italian. doi:10.1702/ 3080.30722

14. Teo KY, Ang SY, Bian L, et al. Evaluating the effectiveness of silicone multilayer foam dressing in preventing heel pressure injury among critically ill patients in Singapore [online]. Wound Pract Res. 2018;26:76-82.

15. Ramundo J, Pike C, Pittman J. Do prophylactic foam dressings reduce heel pressure injuries? J Wound Ostomy Continence Nurs. 2018;45(1):75-82. doi:10.1097/WON.0000000000000400

16. Reid K, Ayello EA, Alavi A. Pressure ulcer prevention and treatment: use of prophylactic dressings. Chron Wound Care Manage Res. 2016;3:117-121. doi:10.2147/CWCMR.S78422

17. Santamaria N, Liu W, Gerdtz M, et al. The cost-benefit of using soft silicone multilayered foam dressings to prevent sacral and heel pressure ulcers in trauma and critically ill patients: a within-trial analysis of the border trial. Int Wound J. 2015;12(3):344-350. doi:10.1111/ iwj. 12160

18. Behnammoghadam M, Paymard A, Salehian T, et al. The effect of local olive oil on prevention of pressure ulcers in patients hospitalized in the intensive care unit of Shahid Beheshti Hospital, Yasuj: a double-blind randomized clinical trial. JAP. 2017;7(4):54-61.
19. Huang TH, Wang PW, Yang SC, Chou WL, Fang JY. Cosmetic and therapeutic applications of fish oil's fatty acids on the skin. Mar Drugs. 2018;16(8):E256. doi:10.3390/md16080256

20. Aloweni F, Lim ML, Chua TL, Tan SB, Lian SB, Ang SY. A randomised controlled trial to evaluate the incremental effectiveness of a prophylactic dressing and fatty acids oil in the prevention of pressure injuries. Wound Pract Res. 2017;25(1):25-34.

21. Pressure ulcer staging; 2019. Available from: https://www.npuap.org/ resources/educational-and-clinical-resources/npuap-pressure-injurystages/. Accessed January 13, 2020.

22. Abbas Ali Madadi Z, Zeighami R, Azimian J, Javadi A. The effect of topical olive oil on prevention of bedsore in intensive care units patients. Int J Res Med Sci. 2015;3(9):2342-2347.

23. Elahi N, Mojdeh S, Poordad A. The effect of fish oil on improvement of first stage bed sore. Iran J Nurs Midwifery Res. 2012;17 (4):313-317.

24. Nagai K, Matsumaru K, Hirai I, Takae Y, Andoh K. New therapy using omega-3-acid ethyl esters for decubitus ulcers and stasis dermatitis: a case report. Iran Red Crescent Med J. 2014;16(12):e19500. doi:10.5812/ircmj. 19500

25. Lupiañez-Perez I, Uttumchandani SK, Morilla-Herrera JC, et al. Topical olive oil is not inferior to hyperoxygenated fatty aids to prevent pressure ulcers in high-risk immobilised patients in home care. Results of a multicentre randomised triple-blind controlled non-inferiority trial. PLoS One. 2015;10(4):e0122238.

26. Lupiáñez-Pérez I, Morilla-Herrera JC, Ginel-Mendoza L, et al. Effectiveness of olive oil for the prevention of pressure ulcers caused in immobilized patients within the scope of primary health care: study protocol for a randomized controlled trial. Trials. 2013;14:348. doi:10.1186/1745-6215-14-348

27. Karimi Z, Behnammoghadam M, Rafiei H, et al. Impact of olive oil and honey on healing of diabetic foot: a randomized controlled trial. Clin Cosmet Investig Dermatol. 2019;12:347-354. doi:10.2147/ CCID.S198577

\section{Publish your work in this journal}

Clinical, Cosmetic and Investigational Dermatology is an international, peer-reviewed, open access, online journal that focuses on the latest clinical and experimental research in all aspects of skin disease and cosmetic interventions. This journal is indexed on CAS
The manuscript management system is completely online and includes a very quick and fair peer-review system, which is all easy to use. Visit http://www.dovepress.com/testimonials.php to read real quotes from published authors. 